\title{
RG Invariant Higgs Pole Mass from the $O(\hbar)$ Expansion
}

\author{
Chungku Kim \\ Department of Physics, College of Natural Science, Keimyung University, Daegu 705-701, KOREA
}

(Dated: November 10, 2018)

\begin{abstract}
We obtain the one-loop pole mass of the standard model Higgs which is invariant under the beta functions of the MS scheme by following the procedure in which the classical action in the broken symmetry phase was obtaind by adding the $O(\hbar)$ expansion of the vacuum expection value determined from the effective potential of the unbroken phase to the Higgs field $H$.
\end{abstract}

PACS numbers: 11.15.Bt, 12.38.Bx

\section{INTRODUCTION}

The pole mass which plays an important role in the process where the characteristic scale is close to the mass shell[1], was shown to be infrared finite and gauge invariant[2]. The one-loop relation between the scalar quartic coupling constant and other physical quantities such as the Higgs pole mass, Fermi constant and the electromagnetic coupling constant of the standard model(SM) was obtained in the Feynman gauge[3] and the expression of the pole mass of the SM in terms of Lagrangian parameter up to two-loop order was obtained later[4] in general $R_{\xi}$ gauge. The physical quantities such as the pole mass and the beta functions obtained in the minimal subtraction(MS) scheme was used in determining the Higgs mass bound[5] or the vacuum stability analysis[6]. Recently, the renormalization $\operatorname{group}(\mathrm{RG})$ invariance of the pole mass was proved from the RG equation of the two-point Green function[7]. However, the beta functions take different forms when calculated in different renormalization schemes and in many cases, these beta functions are obtained in the unbroken phase with the MS scheme[8]. In this sense, it seems to be necessary to obtain the RG invariant pole mass in the broken phase with the RG functions of the MS scheme. Actually, in Appendix B of [4] where the on-shell renormalization was used, the $\mu$ dependent terms of the one-loop pole mass $M$ of the SM have the a form

$$
M^{2}=m^{2}\left\{1+\hbar\left(\sum_{i, j} c_{i j} \frac{m_{i}^{2}}{m_{j}^{2}} \log \left(\frac{m^{2}}{\mu^{2}}\right)+\text { terms independent of } \mu\right)\right\} .
$$

In this case, in order to satisfy the RG invariance of the pole mass such as

$$
\mu \frac{\partial M^{2}}{\partial \mu}+\beta_{\lambda} \frac{\partial M^{2}}{\partial \lambda}+\beta_{m^{2}} \frac{\partial M^{2}}{\partial m^{2}}=0,
$$

we need the one-loop beta function containing the ratios of the masses as

$$
\beta_{m^{2}}=-2 \hbar m^{2} \sum_{i, j} c_{i j} \frac{m_{i}^{2}}{m_{j}^{2}}
$$

By noting that the $(\text { mass })^{2}$ is given by the multiplication of the vacuum expectation value(VEV) and the coupling constants, this implies that $\beta_{m^{2}}$ contains the ratio of the coupling constants. It is evident that the $\beta_{m^{2}}$ obtained in the MS scheme given in Eq.(16) below do not contain this kind of term. Recently, we have shown that[9] if we take the classical action of the broken symmetry phase by adding the vacuum expectation value determined from the effective potential to the scalar field in the unbroken phase, the resulting effective action of the broken symmetry phase is invariant under the RG equation with the beta functions of the unbroken phase and the resulting pole mass is RG invariant[7]. In this paper, we will calculate the one-loop SM Higgs pole mass in terms of the Lagrangian parameters in the Landau gauge which is invariant under the beta functions in the MS scheme by following the procedure used in [7] in case of the neutral scalar theory. In Sec.II, we will determine the vacuum expectation value(VEV) of the SM by using the effective potential obtained in the Landau gauge[10]. Then we calculate the two-point Green function of the SM by using the classical action of the broken symmetry phase obtained by substituting the field with the vanishing VEV. As a result, we can check that the tadpole contribution cancels exactly with the tadpole counterterm[11] and the resulting Higgs pole mass was invariant under the beta function of the MS scheme. In Sec. III, we give some discussion and conclusion. 


\section{RG INVARIANT SM HIGGS POLE MASS AND THE $O(\hbar)$ EXPANSION}

Consider the gauge-scalar part of the SM in the unbroken phase with the bare classical action given by

$$
I_{B}=\int d^{D} x\left[\left|D_{\mu} \Phi_{B}\right|^{2}+m_{B}^{2} \Phi_{B}^{\dagger} \Phi_{B}+\frac{1}{6} \lambda_{B}\left(\Phi_{B}^{\dagger} \Phi_{B}\right)^{2}+L_{g f}+L_{g h}\right]
$$

where $L_{g f}$ and $L_{g h}$ are gauge fixing and the ghost terms and $D \equiv 4-2 \varepsilon$ is the dimension of the space-time and $\Phi$ is the scalar doublet

$$
\Phi=\left(\begin{array}{c}
\Phi^{+} \\
\frac{1}{\sqrt{2}}\left(H+\Phi_{0}\right)
\end{array}\right)
$$

with

$$
D_{\mu} \Phi_{B}=\left(\partial_{\mu}-\frac{i}{2} g_{B} \vec{\tau} \cdot \vec{W}_{B \mu}-\frac{i}{2} g_{B}^{\prime} B_{B \mu}\right) \Phi_{B}
$$

The bare quantities can be expressed in terms of the renormalized quantities such as

$$
m_{B}=m\left(1+\delta_{m^{2}}\right), \lambda_{B}=\mu^{2 \varepsilon} \lambda\left(1+\delta_{\lambda}\right) \text { and } \Phi_{B}=\Phi\left(1+\delta_{\phi}\right),
$$

and similarly for $g_{B}, g_{B}^{\prime}, W_{B}$ and $B_{B}$. Then the effective action that give the 1PI Feynman diagrams with the external Higgs lines is given by

$$
\exp \left(-\frac{1}{\hbar} \Gamma(\phi)\right)=\int D \Phi \exp \left[-\frac{1}{\hbar}\left\{I_{B}(H+\phi)+J \Phi\right\}\right]
$$

and the corresponding effective potential $V(\phi)$ can be obtained by putting $\phi$ as a constant. In case of the broken symmetry phase where the renormalized mass $m^{2}<0$, the Higgs field $\mathrm{H}$ develops a VEV $v$ which satisfies

$$
\left[\frac{\delta V(\phi)}{\delta \phi}\right]_{\phi=v}=0
$$

from which we can determine $v$ as power series in $\hbar$;

$$
v=v_{0}+\sum_{n=1}^{\infty} \hbar^{n} v_{n}\left(v_{0}^{2}=-\frac{6 m^{2}}{\lambda}\right) .
$$

Now, we choose the classical action with the Higgs field which do not have VEV by substituting

$$
\Phi=\left(\begin{array}{l}
\Phi^{+} \\
\frac{1}{\sqrt{2}}\left(H+v+\Phi_{0}\right)
\end{array}\right)
$$

into Eq.(4) and with the counterterms obtained in the unbroken phase of the MS scheme. By this choice, $H$ has a vanishing VEV and hence the tadpole diagrams cancel automatically[7, 11] as compared to the usual on shell scheme[12] where the counterterms of parameters of the classical action are determined from the no tadpole condition. The resulting classical action contains not only the order $\hbar^{n}(n>1) \frac{1}{\varepsilon}$ divergent MS counterterms but also finite counterterms coming from the $\sum_{n=1}^{\infty} \hbar^{n} v_{n}$ given in Eq.(10). From Eq.(8), the effective action in the broken symmetry phase $\Gamma_{B S}(\phi)$ is related to $\Gamma(\phi)$ as

$$
\Gamma_{B S}(\phi)=\Gamma(\phi+v) .
$$

It was shown that $\Gamma_{B S}(\phi)$ satisfies the RG equation with the MS beta functions in the unbroken symmetry[9] and the resulting pole mass is $\mathrm{RG}$ invariant[7].

Now, let us apply the above procedure to SM and obtain the RG invariant Higgs mass. For this purpose, we start from the renormalized one-loop effective potential obtained in Landau gauge[10] in which the Goldstone bosons are massless and the ghost terms couple only to the gauge bosons. The one-loop effective potential is given by $V(\phi)=V_{0}(\phi)+\hbar V_{1}(\phi)$ where

$$
V_{0}(\phi)=\frac{1}{2} m^{2} \phi^{2}+\frac{1}{24} \lambda \phi^{4}
$$


and

$$
(4 \pi)^{2} V_{1}(\phi)=\frac{1}{4} H^{2}\left(\overline{\ln } H-\frac{3}{2}\right)+\frac{3}{4} G^{2}\left(\overline{\ln } G-\frac{3}{2}\right)+\frac{3}{2} W^{2}\left(\overline{\ln } W-\frac{5}{6}\right)+\frac{3}{4} Z^{2}\left(\overline{\ln } Z-\frac{5}{6}\right),
$$

where

$$
H=m^{2}+\frac{\lambda}{2} \phi^{2}, G=m^{2}+\frac{\lambda}{6} \phi^{2}, W=\frac{g^{2}}{4} \phi^{2}, Z=\frac{\left(g^{2}+g^{\prime 2}\right)}{4} \phi^{2} \text { and } \overline{\ln } H=\ln \frac{X}{4 \pi \mu^{2}}+\gamma,
$$

and satisfies the RG equation with the MS beta functions

$$
\begin{aligned}
\beta_{\lambda} & =\mu \frac{d \lambda}{d \mu}=\frac{\hbar}{(4 \pi)^{2}}\left(4 \lambda^{2}-9 \lambda g^{2}-3 \lambda g^{\prime 2}+\frac{9}{4} g^{\prime 4}+\frac{9}{2} g^{2} g^{\prime 2}+\frac{27}{4} g^{4}\right)+\cdots, \\
\beta_{m^{2}} & =\frac{\mu}{m^{2}} \frac{d m^{2}}{d \mu}=\frac{\hbar}{(4 \pi)^{2}}\left(2 \lambda-\frac{9}{2} g^{2}-\frac{3}{2} g^{\prime 2}\right)+\cdots, \\
\gamma & =\frac{\mu}{\phi} \frac{d \phi}{d \mu}=\frac{\hbar}{(4 \pi)^{2}}\left(-\frac{9}{4} g^{2}-\frac{3}{4} g^{\prime 2}\right)+\cdots
\end{aligned}
$$

By using Eq.(9) and (13)-(15), we obtain

$$
v_{1}=\frac{1}{16 \pi^{2} \lambda v_{0}}\left[\frac{3 \lambda}{2} m_{H}^{2}\left\{\overline{\ln }\left(m_{H}^{2}\right)-1\right\}+\frac{9 g^{2}}{2} m_{W}^{2}\left\{\overline{\ln }\left(m_{W}^{2}\right)-\frac{1}{3}\right\}+\frac{9\left(g^{2}+g^{\prime 2}\right)}{4} m_{Z}^{2}\left\{\overline{\ln }\left(m_{Z}^{2}\right)-\frac{1}{3}\right\}\right] .
$$

where $m_{H}^{2} \equiv m^{2}+\frac{\lambda}{2} v_{0}^{2}=-2 m^{2}, m_{W}^{2} \equiv \frac{1}{4} g^{2} v_{0}^{2}=-\frac{3 g 2}{2 \lambda} m^{2}$ and $m_{Z}^{2} \equiv \frac{1}{4}\left(g^{2}+g^{\prime 2}\right) v_{0}^{2}=-\frac{3\left(g^{2}+g^{\prime 2}\right)}{2 \lambda} m^{2}$. Now, the one-loop two-point Green function $\Pi\left(p^{2}\right)$ is given by

$$
\Pi\left(p^{2}\right)=p^{2}+m^{2}+\frac{\lambda}{2} v_{0}^{2}+\hbar \Pi^{(1)}\left(p^{2}\right),
$$

where $\Pi^{(1)}\left(p^{2}\right)$ is given by

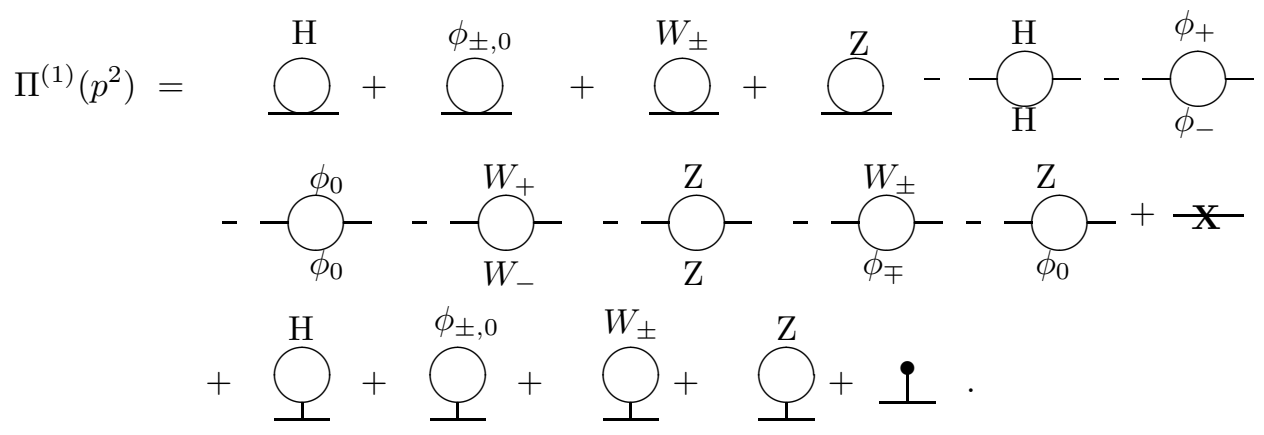

By substituting Eqs.(10) and (11) into Eq.(4), we can obtain the counterterms for the tadpole as

$$
\text { - }=m^{2} v+\frac{\lambda}{6} v^{3}+m^{2} v\left(\delta_{m^{2}}+2 \delta_{\phi}\right)+\frac{\lambda}{6} v^{3}\left(4 \delta_{\phi}+\delta_{\lambda}\right) \simeq \hbar\left\{-2 m^{2} v_{1}+m^{2} v_{0}\left(\delta_{m^{2}}^{(1)}-2 \delta_{\phi}^{(1)}-\delta_{\lambda}^{(1)}\right)\right\},
$$

and that for the two-point Green function

$$
\mathbf{X}=\frac{\lambda}{2}\left(v^{2}-v_{0}^{2}\right)+2 p^{2} \delta_{\phi}+m^{2}\left(\delta_{m^{2}}+2 \delta_{\phi}\right)+\frac{\lambda}{2} v^{2}\left(4 \delta_{\phi}+\delta_{\lambda}\right) \simeq \hbar\left\{\lambda v_{0} v_{1}+2 p^{2} \delta_{\phi}+m^{2}\left(\delta_{m^{2}}^{(1)}-10 \delta_{\phi}^{(1)}-3 \delta_{\lambda}^{(1)}\right)\right\} .
$$

By using the one-loop MS counterterms in the Landau gauge

$$
\begin{aligned}
& \delta_{\phi}^{(1)}=\frac{\hbar}{16 \pi^{2} \varepsilon}\left\{\frac{9 g^{2}}{8}+\frac{3 g^{\prime 2}}{8}\right\}, \delta_{m^{2}}^{(1)}=\frac{\hbar}{16 \pi^{2} \varepsilon}\left\{\lambda-\frac{9 g^{2}}{4}-\frac{3 g^{\prime 2}}{4}\right\}, \\
& \delta_{\lambda}^{(1)}=\frac{\hbar}{16 \pi^{2} \varepsilon}\left\{2 \lambda-\frac{9 g^{2}}{2}-\frac{3 g^{\prime 2}}{2}+\frac{9 g^{4}}{4 \lambda}+\frac{9\left(g^{2}+g^{\prime 2}\right)^{2}}{8 \lambda}\right\},
\end{aligned}
$$


we can see that the tadpole counterterm - cancels with the tadpole diagrams given in last line of Eq.(19) completely as

$$
\frac{\lambda}{2} v_{0} A\left(m_{H}\right)+(3-2 \varepsilon)\left\{\frac{g^{2}}{2} v_{0} A\left(m_{W}\right)+\frac{\left(g^{2}+g^{\prime 2}\right)}{4} v_{0} A\left(m_{Z}\right)\right\}+\bullet=0
$$

where

$$
A(m)=\int \frac{d^{D} q}{(2 \pi)^{D}} \frac{1}{q^{2}+m^{2}}=-\frac{m^{2}}{16 \pi^{2}}\left(\frac{1}{\varepsilon}+1-\overline{\ln } m^{2}\right)
$$

Then the one-loop two-point Green function $\Pi^{(1)}\left(p^{2}\right)$ can be written as

$$
\begin{aligned}
\Pi^{(1)}\left(p^{2}\right)= & \lambda v_{0} v_{1}+2 p^{2} \delta_{\phi}+m^{2}\left(\delta_{m^{2}}^{(1)}-10 \delta_{\phi}^{(1)}-3 \delta_{\lambda}^{(1)}\right) \\
& +\frac{\lambda}{2} A\left(m_{H}\right)+(3-2 \varepsilon)\left\{\frac{g^{2}}{2} A\left(m_{W}\right)+\frac{\left(g^{2}+g^{\prime 2}\right)}{4} A\left(m_{Z}\right)\right\} \\
& -\frac{1}{2} \lambda^{2} v_{0}^{2} B_{0}\left(p, m_{H}, m_{H}\right)-\frac{1}{6} \lambda^{2} v_{0}^{2} B_{0}(p, 0,0)-\frac{1}{4} g^{4} v_{0}^{2} F\left(p, m_{W}\right) \\
& -\frac{1}{8}\left(g^{2}+g^{\prime 2}\right)^{2} v_{0}^{2} F\left(p, m_{Z}\right)-\frac{1}{2} g^{2} G\left(p, m_{W}\right)-\frac{1}{4}\left(g^{2}+g^{\prime 2}\right) G\left(p, m_{Z}\right),
\end{aligned}
$$

where $B_{0}\left(p, m_{1}, m_{2}\right)$ is the one-loop momentum integrals of Passarino and Veltman[13]

$$
B_{0}\left(p, m_{1}, m_{2}\right)=\int \frac{d^{D} q}{(2 \pi)^{D}} \frac{1}{\left(q^{2}+m_{1}^{2}\right)\left((p+q)^{2}+m_{2}^{2}\right)}=\frac{1}{16 \pi^{2}}\left\{\frac{1}{\varepsilon}+\int_{0}^{1} \overline{\ln }\left(\alpha m_{1}^{2}+(1-\alpha) m_{2}^{2}+\alpha(1-\alpha) p^{2}\right) d \alpha\right\},
$$

and $F(p, m)$ and $G(p, m)$ are defined by

$$
\begin{aligned}
F(p, m)= & \left.\frac{p^{4}}{4 m^{4}}\left\{B_{0}(p, m, m)-2 B_{0}(p, m, 0)+B_{0}(p, 0,0)\right\}+\left(\frac{p^{2}}{m^{2}}+\frac{1}{2}\right)\left\{B_{0}(p, m, m)\right\}-B_{0}(p, m, 0)\right\}-\frac{1}{2 m^{2}} A(m) \\
& +\left(\frac{5}{2}-2 \varepsilon\right) B_{0}(p, m, m)
\end{aligned}
$$

and

$$
\left.G(p, m)=\frac{p^{4}}{m^{2}}\left\{B_{0}(p, m, 0)-B_{0}(p, 0,0)\right\}+p^{2}\left\{2 B_{0}(p, m, 0)\right\}-\frac{A(m)}{m^{2}}\right\}+m^{2} B_{0}(p, m, 0)+A(m) .
$$

Note that the $\frac{1}{\varepsilon}$ divergent terms of the two-point Green function cancel with those from the counterterms for the two-point Green function ( $\mathbf{X}$ ) and only the contribution of the finite term $\lambda v_{0} v_{1}$ of $\mathbf{X}$ remains.

Now, the Higgs pole mass $M^{2}$ is defined to be the pole of the two-point Green function given in Eq.(18) so that

$$
\left[\Pi_{R}\left(p^{2}\right)\right]_{p^{2}=-M^{2}}=0
$$

It turns out that the functions $B_{0}(p, 0,0)$ and $B_{0}(p, m, 0)$ which were present in the functions $F(p, m)$ and $G(p, m)$ vanishes in the pole mass $M^{2}$ and we obtain

$$
\begin{aligned}
M^{2}= & -2 m^{2}+\hbar\left[\Pi_{R}^{(1)}\left(p^{2}\right)\right]_{p^{2}=2 m^{2}} \\
= & m_{H}^{2}+\hbar m^{2}\left[\left(4 \lambda-3 g^{2}-g^{\prime 2}+\frac{9 g^{4}}{2 \lambda}+\frac{9\left(g^{2}+g^{\prime 2}\right)^{2}}{4 \lambda}\right) \ln \left(\frac{m_{H}^{2}}{\mu^{2}}\right)+3 \lambda b\left(m_{H}\right)+\left(-2 g^{2}+\frac{9 g^{4}}{2 \lambda}+\frac{2 \lambda}{3}\right) b\left(m_{W}\right)\right. \\
& +\left\{-\left(g^{2}+g^{\prime 2}\right)+\frac{9\left(g^{2}+g^{\prime 2}\right)^{2}}{4 \lambda}+\frac{\lambda}{3}\right\} b\left(m_{Z}\right)-2 \lambda\left\{\ln \left(\frac{m_{H}^{2}}{\mu^{2}}\right)-1\right\}-\left(g^{2}+\frac{9 g^{4}}{2 \lambda}\right)\left\{\ln \left(\frac{m_{W}^{2}}{\mu^{2}}\right)-1\right\} \\
& \left.-\left(\frac{\left(g^{2}+g^{\prime 2}\right)}{2}+\frac{9\left(g^{2}+g^{\prime 2}\right)^{2}}{4 \lambda}\right)\left\{\ln \left(\frac{m_{Z}^{2}}{\mu^{2}}\right)-1\right\}\right],
\end{aligned}
$$

where

$$
b(m)=2 \sqrt{4 z-1} \tan ^{-1}\left(\frac{1}{\sqrt{4 z-1}}\right)+\ln (z)-2\left(z \succeq \frac{1}{4}\right)
$$

with $z \equiv \frac{m^{2}}{m_{H}^{2}} \succeq \frac{1}{4}$ for $m^{2}=m_{H}^{2}, m_{W}^{2}$ and $m_{Z}^{2}$. One can check that the pole mass given in Eq.(30) satisfies the RG equation for the pole mass (Eq.(4)) with the MS beta function $\beta_{m^{2}}$ given in Eq.(16). 


\section{DISCUSSIONS AND CONCLUSIONS}

In thisn paper, we have calculated the one-loop pole mass of the standard model(SM) Higgs in the Landau gauge which is invariant under the beta functions of the MS scheme. By adding the $O(\hbar)$ expansion of the VEV obtained from the effective potential of the unbroken phase to Higgs field $H$, we obtain the classical action in the broken symmetry phase which contains both the $\frac{1}{\varepsilon}$ divergent counterterms coming from the MS scheme and the finite counterterms from the $\hbar$ expansion VEV. The tadpole diagrams vanishes automatically and the resulting pole mass was invariant under the RG equation with the beta functions of the MS scheme obtained in the unbroken phase. This procedure to obtain the RG invariant pole mass in the broken symmetry phase can be extended to the case of the gauge bosons. Then the scalar quartic coupling constant $\lambda$ can be expressed by these pole masses which can provide another initial condition for $\lambda$ in contrast to the previous result[3] where $\lambda$ was obtained in terms of the Higgs mass, Fermi constant and the electromagnetic coupling constant and this is under the investigation.

[1] S. Narison, Phys. Lett. B216,191 (1989).

[2] A. S. Kronfeld, Phys. Rev. D58 , 051501 (1998).

[3] A. Sirlin and R. Zucchini, Nucl. Phys. B266,389 (1986).

[4] F. Jegerlehner, M. Yu. Kalmykov and O. L. Veretin, Nucl. Phys. B641, 285 (2002); B658, 49 (2003).

[5] P. Kielanowski and S. R. Juarez W, Phys. Rev. D72 , 096003(2005).

[6] M. Lindner, M.Sher and W. Zaglauer, Phys. Lett. B228,139 (1989), G. Altarelli and G.Isidori, ibid, B337,141 (1994), J.A.Casas, J.R.Espinosa and M.Quiros, ibid, B382,374 (1996).

[7] C. Kim, J. Korean Phys. Soc. 62,1097 (2013).

[8] A.V. Bednyakov, A.F. Pikelner, V. N. Velizhanin, JHEP, 1301, 017, (2013), K. G. Chetyrkin, M. F. Zoller, JHEP, 1304 091.

[9] C. Kim, J. Kor. Phys. Soc. 59, 2993 (2011).

[10] A. C.Ford, D.R.T.Jones, I.Jack. Nucl. Phys. B387,373 (1992), Erratum ibid B504,551 (1997).

[11] J. C. Taylor, Gauge Theories of Weak Interactions (Cambridge Press, Cambridge, 1976).

[12] A. Sirlin, Phys. REv. D, 22 , 2695 (1980), M. Bohm, W. Hollik, H. Spiesbeer, Fortschr. Phys. 34687 (1986).

[13] G. Passarino and M. Veltman, Nucl. Phys. B 160, 151 (1979). 